\title{
Humanité et sérieux dans le quotidien d’une généraliste
}

\section{Jean Martin}

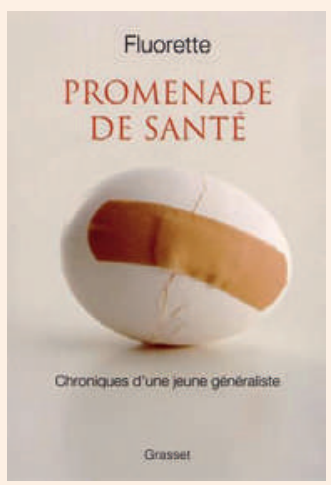

Fluorette

Promenade de santé

Chroniques d'une jeune généraliste

Paris: Bernard Grasset; 2014 335 pages. 16.90 Euros. ISBN 978-2-2468-0666-0
Logiquement, la jeune génération médicale française communique par les moyens modernes, blogs notamment. «Promenade de santé» est issu pour une bonne part de celui de Fluorette, 35 ans environ. Après avoir vécu et suivi études, externat et internat pas loin de la mer (qui lui manque), elle a fait des remplacements puis, pour des raisons conjugales, s'est installée en cabinet de groupe en Alsace (elle évoque les difficultés rencontrées avec des patients parlant allemand ou patois). Les plus de 80 vignettes de l'ouvrage sont dans la même veine que «Juste après dresseuse d'ours», de Jaddo [1]. On pense aussi à «Le Chœur des femmes» de Martin Winckler [2], dont l'héroïne-narratrice décrit son activité dans un centre de soins de premier recours. L'intérêt pour le métier, le dynamisme, les satisfactions et joies - de fréquence et intensité variables, sont de la même veine, mais aussi les difficultés et moments de déprime, assez nombreux. On est tenté de comparer ces livres et ce n'est pas une mauvaise idée à mon sens - je recommande la lecture des trois. Commentaire d'un confrère sur le site egora.fr («Les médecins prennent la parole»): «Je suis les blogs de Fluorette et de Jaddo. Elles sont les représentantes et la quintessence d'une génération de femmes qui arrivent avec une approche intelligente de l'exercice médical: moins d'actes, super formation...»

Fluorette parle d'abord de ses études de médecine et de ses stages hospitaliers. Notant par exemple: «A l'hôpital, je n'appréciais pas la façon dont on traitait parfois les patients, sans respect pour leur pudeur, en s'indignant parfois qu'ils puissent avoir un avis sur leur propre cas»... Ensuite, les courts récits de «Promenade de santé» sont pour l'essentiel des descriptions de moments de l'activité quotidienne, en cabinet ou lors de visites à domicile (qui semblent encore nombreuses).

«'écris, dit-elle, pour tenter de décharger mes épaules du poids de mes journées et soigner mes insomnies. Pour avoir des avis sur certaines situations et exorciser mes angoisses.» Mais il y a aussi des occasions de ressourcement dans la nature, observant un groupe de cigognes ou d'oies sauvages.

De manière encore plus appuyée que ses consœurs des livres susmentionnés, elle insiste sur une pratique fondée sur les preuves (EBM), aussi en médecine de premier recours; ceci tout en mettant l'accent sur la qualité de la relation, l'écoute. Toujours respectueuse du patient et du temps qu'elle lui consacre. Illustrant une pratique moderne, scientifique et relationnelle. Elle entend limiter la polymédication si fréquente «'aime tenter d'arrêter des catastrophes médicamen- teuses ancestrales». Elle refuse les renouvellements de prescription sur un coin de bureau, voire par la secrétaire, insiste sur les règles de bonne pratique comme «Les antibiotiques ne sont pas automatiques» - selon le titre d'une campagne faite en France. Sans dureté, elle met certains consultants devant leur responsabilité quant au bon usage des ressources du système de santé. Sauf urgence, elle ne voit pas les personnes qui n'acceptent pas consultation et examen complets, qui veulent que le médecin se limite à leur plainte immédiate (encore que, sur ce point, Fluorette semble moins ferme à la fin de l'ouvrage qu'au début).

Elle ne transige pas, à la lire, sur les principes d'une activité médicale de qualité, en fonction des connaissances actuelles, et économique - qui ne se laisse pas tenter par les artifices permettant d'élever indûment son revenu. «Financièrement, c'est plus difficile que si j'acceptais d'aller contre la médecine en laquelle je crois, contre les principes qui me semblent importants». Ne cachant pas comment elle a été surprise, parfois secouée, par les lenteurs et complications répétées des relations avec les administrations, notamment celle de l'assurance-maladie. Dans l'actualité, elle est très critique de l'action de la Ministre française de la santé Marisol Touraine, à qui elle s'adresse à propos de la manière dont elle dit vouloir s'intéresser à la médecine générale et aux «déserts médicaux» («avec cet aplomb dont seuls les menteurs sont capables quand ils savent que ce qu'il racontent est faux mais qu'ils veulent convaincre»).

Ouvrage tonique et crédible, bien écrit, plein d'humanité et de sérieux (peu ou pas de plaisanteries, ni dans son activité ni dans la manière dont elle la décrit), d'une professionnelle qui a choisi la médecine, et particulièrement la médecine générale, parce qu'elle aime les gens. Mettant en évidence, malgré les beautés du métier, les frustrations qui sont les siennes et celles de beaucoup de généralistes; l'impression d'être considérés comme des confrères de seconde zone, géographiquement, professionnellement, sociétalement. On veut espérer que cet ouvrage n'échappera pas, en haut lieu, à l'attention des enseignants des facultés, des responsables des associations professionnelles, des planificateurs et autres conseillers techniques.

1 Martin J. Le récit qui donne envie de devenir médecin généraliste (à propos de «Juste après dresseuse d'ours», de Jaddo). Bull Méd Suisses. 2013;94(40):1524-5.

2 Martin J. Relation de soin médecin-patiente dans les domaines liés à la sexualité (à propos de «Le Choeur des femmes», de Martin Winckler). Bull Méd Suisses. 2013; 94(10):401-2. 